\title{
SUBAMOSTRAGEM COM UNIDADES PRIMÁRIAS DE TAMANHOS DESIGUAIS APLICADA EM INVENTÁRIOS DE FLORESTAS PLANTADAS \\ DE Eucalyptus grandis
}

\author{
Sérgio Aparecido Ignácio ${ }^{1}$ \\ Dartagnan Baggio Emerenciano ${ }^{2}$ \\ Sylvio Péllico Netto ${ }^{3}$ \\ Carlos Roberto Sanquetta ${ }^{4}$
}

\begin{abstract}
RESUMO
O objetivo deste estudo foi avaliar a precisão e eficiência de dois processos de subamostragem com unidades primárias de tamanhos desiguais, em um povoamento de Eucalyptus grandis em regime de alto fuste e plantio com semente, localizado no município de Mogi Guaçu, no Estado de São Paulo, pertencente a International Paper do Brasil Ltda. Trabalhou-se com as informações das medições realizadas pela empresa, envolvendo talhões com idade entre 6 e 8 anos. Os dois processos de subamostragem testados foram: Processo I - unidades primárias selecionadas com probabilidades iguais - estimador tendencioso por índice em relação ao tamanho do talhão e processo II - unidades primárias selecionadas com probabilidades proporcionais ao tamanho do talhão - estimador não tendencioso. Constatou-se que o Processo I foi superior ao Processo II, com uma eficiência relativa de 75,83\%, implicando na obtenção de intervalos de confiança mais estreitos para a média e total.

Palavras-chave: inventário florestal; processos de amostragem; erro de amostragem, eficiência relativa
\end{abstract}

\section{SUB-SAMPLING WITH UNEQUAL SIZE PRIMARY UNITS APPLIED TO INVENTORIES OF Eucalyptus grandis FOREST STANDS}

\begin{abstract}
This study assessed the precision and efficiency of two sub-sampling processes with unequal size primary units in one Eucaliptus grandis stand in managed clear-cut regimes, located in Mogi Guaçu municipalitie, in the State of São Paulo, belonging to International Paper do Brasil Ltda. The study died use of measurements performed by the company in the Eucalyptus grandis stand, involving 6 and 8 year's compartments. The two tested subsampling were: Processe I - selected primary units with equal probability-biased estimator ratio to size and Processe II - selected primary units with probability proportional to size - unbiased estimator. By comparing them regarding their relative efficiency, it was verified that the process of sub-sampling I showed the best result when compared to the process II, with relative efficiency of $75,83 \%$, with smaller confidence intervals for the means and total.

Key-words: Forest inventory; sampling process; sampling error; relative efficiency

\footnotetext{
Estatístico. MSc., Professor Titular de Probabilidade e Estatística, PUC-PR e Doutor em Eng. Florestal, UFPR; Rua Macapá 1129 - 82629110, Curitiba, PR; e-mail: sergioai@pr.gov.br.

2 Eng. Florestal, MSc., Dr., Professor do Departamento de Ciências Florestais, UFPR; Rua Eduardo Geronasso - 82510-280, Curitiba, PR; e-mail: darta@nc.ufpr.br.

${ }^{3}$ Eng. Florestal, MSc., Dr., Professor Senior do Departamento Ciências Florestais, UFPR; Rua Rocha Pombo 791 - 80530-290, Curitiba, PR; e-mail: pellico@rla13.pucpr.br.

Eng. Florestal, MSc., Dr., Professor do Departamento de Pós-Graduação em Eng. Florestal, UFPR; Rua Mamoré 1161 - 80810-080, Curitiba, PR; e-mail: sanqueta@floresta.ufpr.br.
} 


\section{INTRODUÇÃO}

O processo de amostragem a ser aplicado dentro de cada estrato de uma população florestal constitui um dos importantes componentes do inventário florestal contínuo (Péllico Netto e Brena, 1997). As áreas reflorestadas, em geral, são planejadas administrativamente e são formadas por unidades mínimas (talhões), de tamanhos variáveis, denominadas unidades primárias de amostragem. Essas unidades permitem ao técnico utilizá-las como unidades de planejamento da produção florestal, dentro das quais é efetuado um segundo estágio da amostragem, geralmente denominado de subamostragem, em que são alocadas as parcelas, denominadas unidades secundárias de amostragem. Do ponto de vista estatístico, fica caracterizado nessa estrutura um processo de amostragem em dois estágios com unidades primárias de tamanhos desiguais.

Segundo Péllico Netto (1996), as discussões sobre esse tema não têm sido realizadas por dois motivos básicos: primeiro, porque se efetua uma baixa intensidade de amostragem dentro do povoamento, de tal forma a não incluir os talhões como partes integrantes dos estimadores estatísticos, e, segundo, porque se utiliza apenas uma estrutura aleatória dentro do povoamento, em que as unidades amostrais se distribuem dentro dos talhões, mas sem que o processo se caracterize como dois estágios para a alocação da amostragem e para o cálculo da média e variância da distribuição amostral de médias.

Os conceitos de subamostragem com unidades primárias de tamanhos desiguais foram inicialmente utilizados por Hansen e Hurwitz (1943). Os autores demonstraram que o uso de probabilidades de seleção desiguais dentro de um estrato pode fornecer estimadores mais eficientes para a média e o total populacional do que os estimadores obtidos pelo uso de probabilidades iguais de seleção.

Cochran (1953) comparou os diferentes processos de subamostragem com unidades primárias de tamanhos desiguais, com reposição, e desenvolveu as fórmulas matemáticas para unidades primárias selecionadas com probabilidades iguais, unidades primárias selecionadas com probabilidades proporcionais a uma medida de grandeza estimada, unidades primárias selecionadas com probabilidades proporcionais ao tamanho e unidades primárias selecionadas com probabilidades proporcionais ao tamanho estimado. $\mathrm{O}$ autor simulou uma pequena população organizada artificialmente e concluiu que os processos cujas unidades primárias são selecionadas com probabilidade proporcional ao tamanho e com probabilidade proporcional a uma medida de grandeza estimada produzem os menores erros de amostragem, sempre que o valor total da variável a ser estimada, em nível de unidade primária, apresentar forte correlação com o valor total da grandeza da variável auxiliar (real ou estimada), e as grandezas apresentarem relativa variabilidade.

Este trabalho teve como objetivo geral avaliar a precisão e eficiência de dois processos de subamostragem com unidades primárias de tamanhos desiguais aplicados em inventários de florestas plantadas de Eucalyptus grandis.

\section{Processos de subamostragem com unidades primárias de tamanhos desiguais}

Apresentam-se a seguir os estimadores para o cálculo do valor médio populacional por unidade secundária $\overline{\bar{Y}}$ e da variância da distribuição amostral de médias, para os dois processos de subamostragem com unidades primárias de tamanhos desiguais.

A derivação das fórmulas foi efetuada considerando-se seleção com reposição para as unidades primárias de amostragem e seleção sem reposição para as unidades secundárias amostradas dentro das unidades primárias selecionadas.

Segundo Cochran (1953), a vantagem de realizar a amostragem com reposição das unidades primárias está na simplicidade da derivação das fórmulas que fornecem as variâncias da distribuição amostral de médias. Em geral, a amostragem com reposição é menos precisa do que a amostragem sem reposição. Entretanto, quando a razão entre o número de unidades primárias selecionadas $(n)$ e o número total de unidades primárias da população $(N)$ é pequeno, a possibilidade de que a mesma unidade apareça mais de uma vez na amostra é reduzida, e a amostragem com reposição é quase equivalente à amostragem 
sem reposição. Porém, se a i-ésima unidade primária for selecionada mais de uma vez, toda a subamostra é reposta e faz-se um novo sorteio independente de $m_{i}$ subunidades, também sem reposição, permitindo assim que a nova amostra contribua para melhorar as estimativas da unidade primária selecionada mais de uma vez.

Sukhatme et al. (1984), ao estudar o processo de subamostragem com probabilidade proporcional ao tamanho da unidade primária, argumenta que as fórmulas derivadas para a amostragem sem reposição influem somente na estimativa da variância entre as unidades primárias de amostragem e a diferença entre as duas formas de seleção, com e sem reposição, não é significante do ponto de vista prático.

\section{Processo I:}

Unidades primárias selecionadas com probabilidades iguais - estimador tendencioso por índice em relação ao tamanho

O estimador do valor médio da população $\overline{\bar{Y}}$ é dado por:

$$
\overline{\bar{y}_{I}}=\frac{\sum_{i=1}^{n}{ }_{M_{i} \bar{y} i}}{\sum_{i=1}^{n} M_{i}}
$$

em que

$\overline{\bar{y}}_{I}=$ média estimada da população, para o processo de subamostragem I;

$\bar{y}_{i}=$ média estimada para a i-ésima unidade primária selecionada; e

$M_{i}=$ número de unidades secundárias potenciais na i-ésima unidade primária selecionada.

Esse é um estimador por índice, uma vez que tanto $o$ numerador quanto $o$ denominador variam de amostra para amostra. Esse estimador tem tendência, mas esta se torna desprezível quando $\mathrm{n}$ é grande.

Segundo (Cochran, 1953), um estimador amostral para a variância da distribuição amostral de médias $S_{\overline{\bar{y}}_{I}}^{2}$ é dado por:

$$
s_{\overline{y_{I}}}=\frac{1}{n}\left[\frac{\sum_{n} \bar{M}^{2}}{n} \cdot \frac{\sum_{i=1}^{n} M_{i}^{2}(1-f 2 i) s_{2 i}^{2}}{m i}+\frac{\sum^{2}{ }^{2}}{\bar{M}^{2}} \frac{\sum_{i=1}^{n} M_{i}^{2}(\bar{y} i-\overline{\bar{y}} I)^{2}}{n-1}\right]
$$

em que

$S_{\overline{\bar{y}}_{I}}^{2}=$ variância da distribuição amostral de médias, obtida com o processo de subamostragem I; e

$f_{1}=\frac{n}{N}=$ fração de amostragem de unidades primárias;

$f_{2 i}=\frac{m_{i}}{M_{i}}=$ fração de amostragem de unidades secundárias, para a i-ésima unidade primária selecionada;

$n=$ número de unidades primárias selecionadas;

$m_{i}=$ número de unidades secundárias amostradas na i-ésima unidade primária selecionada;

$S_{2 i}^{2}=$ variância entre as unidades secundárias amostradas dentro da i-ésima unidade primária selecionada, sendo dada por:

$s_{2 i}^{2}=\frac{1}{m i-1} \sum_{j=1}^{m_{i}}(v i j-\bar{y} i)^{2} ;$

$\bar{M}=$ número médio de unidades secundárias potenciais por unidade primária selecionada na amostra, sendo dado por:

$\bar{M}=\frac{\sum_{i=1}^{n} M_{i}}{n}=\frac{M_{0}}{n} ;$

$M_{0}==$ número total de unidades secundárias potenciais das unidades primárias selecionadas, dado por:

$M_{0}=\sum_{i=1}^{n} M_{i}$.

\section{Processo II:}

Unidades primárias selecionadas com probabilidade proporcional ao tamanho - estimador não tendencioso

Segundo Cochran (1953), se uma amostra de $\mathrm{n}$ unidades primárias (talhões) é escolhida com probabilidade $Z_{i}$ (que 
representa o quociente entre o tamanho do iésimo talhão selecionado e a área total dos talhões selecionados na amostra) e com reposição, a variável $Z_{i}$ é dada por:

$$
Z_{i}=\frac{M_{i}}{M_{0}}
$$

em que

$Z_{i}=$ proporção referente à área do i-ésimo talhão selecionado em relação à área total dos talhões selecionados na amostra;

população $\overline{\bar{Y}}$ é dado por:

$\mathrm{O}$ estimador do valor médio da

$\overline{\bar{y}} I I=\frac{1}{n M 0} \sum_{i=1}^{n} \frac{M_{i} \overline{y_{i}}}{z_{i}}$

em que

$\overline{\bar{y}}_{I I}=$ média estimada da população para o processo de subamostragem II;

$$
\text { Quando } Z_{i}=\frac{M_{i}}{M_{0}} \text {, o estimador dado }
$$

pela expressão (4) se reduz à forma:

$$
\overline{\bar{y}}_{I I}=\frac{\sum_{i=1}^{n} \overline{\bar{y}} i}{n}
$$

A variância da distribuição amostral de médias populacional de $\overline{\bar{y}}_{I I}$ é dada por:

$$
\sigma_{\bar{y}_{I I}}^{2}=\frac{1}{n} \sum_{i=1}^{N} \frac{M_{i}}{M_{0}} \frac{(1-f 2 i) \sigma_{2 i}^{2}}{m_{i}}+\frac{1}{n} \sum_{i=1}^{N} \frac{M_{i}}{M_{0}}\left(\overline{\bar{y}_{i}}-\overline{\bar{\gamma}}\right)^{2}
$$

Considerando que, nesse processo, os estimadores dentro das unidades primárias são autoponderados, Cochran (1953) e Sukhatme et al. (1984) demonstraram que um estimador não tendencioso dessa variância é dado por:

$$
s_{\bar{y}_{I I}}=\frac{1}{n}\left[\frac{1}{(n-1)} \sum_{i=1}^{n}\left(\bar{y}_{i}-\overline{\bar{y}}_{I I}\right)^{2}\right]
$$

\begin{tabular}{llllr} 
Segundo & \multicolumn{2}{c}{ Cochran } & $(1953)$, & o \\
comportamento & relativo do processo de \\
subamostragem & II, em que as unidades
\end{tabular} primárias são selecionadas com probabilidades proporcionais ao tamanho e do processo de subamostragem I, por índice, em que as unidades primárias são selecionadas com probabilidades iguais, depende da relação entre as variâncias de $Y_{i}$ e $M_{i}$. Quando $S_{Y_{i}}^{2}$ é proporcional a $M_{i}^{\beta}$, a estimativa com probabilidades proporcionais ao tamanho é mais precisa que a estimativa por índice, com probabilidades iguais, se $\beta$ é menor que $1 \mathrm{e}$ menos precisa se $\beta$ é maior que 1 .

\section{MATERIAL E MÉTODOS}

O presente trabalho foi utilizado em um plantio de Eucalyptus grandis, em regime de alto fuste, oriundo de semente, pertencente a International Paper do Brasil Ltda., localizada no município de Mogi Guaçu, no Estado de São Paulo. Para o inventário do povoamento, estabeleceram-se parcelas permanentes de $400 \mathrm{~m} 2(20 \mathrm{~m}$ x $20 \mathrm{~m})$, distribuídas aleatoriamente em cada talhão do povoamento, conforme critérios próprios da empresa.

A tabela 1 apresenta as distribuições de parcelas por talhão e para o povoamento estudado, identificando os respectivos talhões em que foram instaladas três ou mais parcelas, a área em hectares, o número potencial de parcelas de tamanho $20 \mathrm{~m} \times 20 \mathrm{~m}\left(M_{i}\right)$, o número de parcelas amostradas aleatoriamente em cada talhão $\left(m_{i}\right)$ e as intensidades amostrais $\left(\frac{m_{i}}{M_{i}}\right)$. 
Tabela 1: Área, número potencial de parcelas $\left(\mathrm{M}_{i}\right)$, número de parcelas lançadas aleatoriamente $\left(m_{i}\right)$ e fração de amostragem $\left(m_{i} / M_{i}\right)$, segundo os talhões pertencentes ao povoamento de Mogi Guaçu - plantio com semente

Table 1: Area, plot potential number $\left(M_{i}\right)$, number of plots randomly chosen $\left(m_{i}\right)$ and sampling fraction $\left(m_{i} / M_{i}\right)$, according to the Mogi Guaçu stands - seed planting

\begin{tabular}{|c|c|c|c|c|c|}
\hline N..$^{o}$ de Ordem & N. ${ }^{o}$ do Talhão & Área(ha) & $M_{i}$ & $m_{i}$ & $m_{i} / M_{i}$ \\
\hline 1 & 1 & 50 & 1250 & 5 & 0,0040 \\
\hline 2 & 2 & 50 & 1250 & 5 & 0,0040 \\
\hline 3 & 3 & 38 & 950 & 5 & 0,0053 \\
\hline 4 & 4 & 51 & 1275 & 5 & 0,0039 \\
\hline 5 & 5 & 50 & 1250 & 5 & 0,0040 \\
\hline 6 & 6 & 48 & 1200 & 5 & 0,0042 \\
\hline 7 & 7 & 29 & 725 & 4 & 0,0055 \\
\hline 8 & 8 & 23 & 575 & 4 & 0,0070 \\
\hline 9 & 9 & 25 & 625 & 4 & 0,0064 \\
\hline 10 & 10 & 29 & 725 & 4 & 0,0055 \\
\hline 11 & 11 & 28 & 700 & 8 & 0,0114 \\
\hline 12 & 12 & 25 & 625 & 4 & 0,0064 \\
\hline 13 & 13 & 25 & 625 & 4 & 0,0064 \\
\hline 14 & 14 & 28 & 700 & 7 & 0,0100 \\
\hline 15 & 15 & 15 & 375 & 4 & 0,0107 \\
\hline 16 & 17 & 29 & 725 & 4 & 0,0055 \\
\hline 17 & 18 & 11 & 275 & 3 & 0,0109 \\
\hline 18 & 19 & 20 & 500 & 4 & 0,0080 \\
\hline 19 & 20 & 10 & 250 & 3 & 0,0120 \\
\hline 20 & 21 & 15 & 375 & 4 & 0,0107 \\
\hline 21 & 22 & 22 & 550 & 4 & 0,0073 \\
\hline 22 & 23 & 16 & 400 & 4 & 0,0100 \\
\hline 23 & 24 & 17 & 425 & 3 & 0,0071 \\
\hline 24 & 25 & 17 & 425 & 4 & 0,0094 \\
\hline 25 & 26 & 9 & 225 & 3 & 0,0133 \\
\hline 26 & 28 & 11 & 275 & 3 & 0,0109 \\
\hline 27 & 40 & 17 & 425 & 3 & 0,0071 \\
\hline 28 & 52 & 24 & 600 & 4 & 0,0067 \\
\hline 29 & 53 & 51 & 1275 & 6 & 0,0047 \\
\hline 30 & 56 & 20 & 500 & 4 & 0,0080 \\
\hline 31 & 57 & 18 & 450 & 3 & 0,0067 \\
\hline 32 & 62 & 46 & 1150 & 5 & 0,0043 \\
\hline 33 & 63 & 15 & 375 & 3 & 0,0080 \\
\hline 34 & 64 & 33 & 825 & 5 & 0,0061 \\
\hline 35 & 65 & 15 & 375 & 3 & 0,0080 \\
\hline 36 & 70 & 24 & 600 & 4 & 0,0067 \\
\hline 37 & 73 & 19 & 475 & 4 & 0,0084 \\
\hline 38 & 74 & 13 & 325 & 3 & 0,0092 \\
\hline 39 & 75 & 32 & 800 & 4 & 0,0050 \\
\hline 40 & 77 & 26 & 650 & 4 & 0,0062 \\
\hline 41 & 79 & 20 & 500 & 3 & 0,0060 \\
\hline 42 & 80 & 29 & 725 & 4 & 0,0055 \\
\hline 43 & 85 & 13 & 325 & 4 & 0,0123 \\
\hline 44 & 86 & 14 & 350 & 4 & 0,0114 \\
\hline TOTAL & - & 1120 & 28000 & 182 & 0,0065 \\
\hline
\end{tabular}

FONTE DOS DADOS BRUTOS: International Paper do Brasil Ltda.

Talhões com menos de três parcelas amostradas não foram considerados neste estudo, pois, para uma parcela, torna-se impossível estimar a variância, e para duas 
parcelas o número de graus de liberdade é um, tornando as estimativas pouco confiáveis. Segundo informações da empresa, a exclusão destes talhões representa menos de $5 \%$ da área total $\mathrm{e}$ do volume de madeira dos povoamentos.

Observa-se na tabela 1 que a intensidade média de amostragem para $o$ povoamento $\left(\frac{m_{i}}{M_{i}}\right)$, foi de 0,0065 , ou $0,65 \%$, representando, em média, uma parcela de 0,04 hectare $(400 \mathrm{~m} 2)$ amostrada para aproximadamente 6 hectares do povoamento. Segundo informações da International Paper do Brasil Ltda., considerando amostragem aleatória simples, essa intensidade amostral garante um erro de amostragem para 0 povoamento de no máximo $\pm 10 \%$, para um nível de confiança $(1-\alpha)$ de $95 \%$. Observa-se, portanto, que o número de parcelas amostradas por talhão é proporcional à sua área, independentemente da variabilidade de cada talhão, critério normalmente utilizado para o dimensionamento de uma amostra.

A empresa forneceu os dados do inventário realizado no povoamento envolvendo a medição dos talhões com idade entre 6 e 8 anos, contendo as seguintes variáveis: região, número do talhão, área do talhão (hectare), número da parcela, material genético (semente ou clone), média aritmética dos diâmetros da parcela a 1,30 metros de altura, com casca $\left(\overline{D A P}_{c / c}\right)$, para árvores com $D A P_{c / c}$ maior ou igual a $2 \mathrm{~cm}$, idade (anos), área basal da parcela, $\mathrm{G}(\mathrm{m} 2 / \mathrm{ha})$, número de árvores da parcela, extrapolado para o hectare, altura dominante média da parcela, $\bar{H}_{\text {dom }}$ (metros) e volume de árvores da parcela, $\mathrm{V}$ (m3/ha).

A população é subdividida em talhões, ou subáreas, considerados como as menores unidades administrativas. Esses talhões variam em tamanho e forma, de acordo com as condições topográficas e com as necessidades administrativas da empresa. A distribuição espacial das árvores nos povoamentos é uniforme, com espaçamentos variáveis de $2 \times 3$ $\mathrm{m}, 2,5 \times 3 \mathrm{~m}$ e $3 \times 3 \mathrm{~m}$.

Em cada parcela foram medidos todos os diâmetros das árvores com $D A P_{c / c}$ maior ou igual a $2 \mathrm{~cm}$.
Com o emprego de um hipsômetro de Blume-Leiss foram medidas as alturas totais das árvores mais altas da parcela, visando definir a média das alturas dominantes $\left(\bar{H}_{d o m}\right)$, empregada como variável independente na equação para estimar o índice de sítio (IS). Considerou-se árvore dominante aquela pertencente ao conjunto das cem árvores de maior diâmetro dentro de um hectare. Selecionaram-se, para parcelas de $400 \mathrm{~m} 2$, as quatro árvores de maior diâmetro como as dominantes.

Para o cálculo das estimativas da área basal e do volume por hectare, para as idades futuras, foram adotadas equações específicas, próprias da empresa. Com a totalização da área basal e do volume por parcela, por meio da soma das áreas transversais e dos volumes por árvore individual, estes foram convertidos para área basal e volume, por hectare. Da mesma forma, para cada parcela, foram obtidas as estimativas do número de árvores por hectare.

As equações utilizadas nos inventários florestais da empresa são as apresentadas a seguir:

a) Índice de sítio

$$
\begin{aligned}
& I S=\bar{H}_{d o m}+\beta_{0}(\ln (7)-\ln (X)) \\
& R^{2}=0,45
\end{aligned}
$$

em que

$I S=$ índice de Sítio = altura dominante projetada para 7 anos (metros);

$\bar{H}_{\text {dom }}=$ média das alturas dominantes da parcela (metros); e

$X=$ idade do inventário (anos).

b) Volume de árvores projetado para idades futuras $\left(\mathrm{m}^{3} / \mathrm{ha}\right)$

$$
\begin{aligned}
V_{f} & =e^{\beta_{0}+\beta_{1}\left(\frac{1}{I S}\right)+\beta_{2}\left(\frac{1}{W}\right)+\beta_{3}\left(\frac{X}{W}\right) \ln (G)+\beta_{4}\left(1-\frac{X}{W}\right)+\beta_{5} I S\left(1-\frac{X}{W}\right)} \\
R^{2} & =0,88
\end{aligned}
$$

em que

$V_{f}=$ volume de árvores da parcela, projetado para idades futuras $\left(\mathrm{m}^{3} / \mathrm{ha}\right)$; 
$I S$ = índice de Sítio (em metros), proveniente da equação (8);

$W=$ idade futura (opcional para idade do inventário, idade atual, idade 7 ou idade de corte (anos));

$X$ = idade do inventário (anos);

$G$ = área basal de árvores da parcela na idade do inventário $\left(\mathrm{m}^{2} / \mathrm{ha}\right)$. Equivale à soma das áreas transversais dos fustes das árvores da parcela, extrapolada para o hectare;

$\beta_{0}=$ constante da equação; $\mathrm{e}$

$\beta_{1}, \beta_{2}, \ldots, \beta_{5}=$ coeficientes da equação.

c) Área basal de árvores projetada para idades futuras $\left(\mathrm{m}^{2} / \mathrm{ha}\right)$

$G_{f}=e^{\left(\frac{X}{W}\right) \ln (G)+\alpha_{1}\left(1-\frac{X}{W}\right)+\alpha_{2} I S\left(1-\frac{X}{W}\right)}$

em que

$G_{f}=$ área basal de árvores da parcela, projetada para idades futuras $\left(\mathrm{m}^{2} / \mathrm{ha}\right)$;

$G=$ área basal de árvores da parcela na idade do inventário $\left(\mathrm{m}^{2} / \mathrm{ha}\right)$. Equivale à soma das áreas transversais dos fustes das árvores da parcela, extrapolada para o hectare;

$X=$ idade do inventário (anos);
$I S$ = índice de Sítio (em metros), proveniente da equação (8);

$W=$ idade futura (opcional para idade do inventário, idade atual, idade 7 ou idade de corte (anos));

$\alpha_{1}, \alpha_{2}=$ coeficientes da equação, sendo dados por:

$\alpha_{1}=\frac{\beta_{4}}{\beta_{3}} ; \mathrm{e}$

$\alpha_{2}=\frac{\beta_{5}}{\beta_{3}}$.

Aplicação da subamostragem com unidades primárias de tamanhos desiguais

Para o povoamento estudado, foi simulada uma subamostragem, com seleção aleatória das unidades primárias, sem reposição. A extração foi feita sem reposição, dado que não seria possível obter uma nova subamostra de unidades secundárias do mesmo talhão e que a repetição da mesma subamostra em nada contribuiria para melhorar as estimativas em nível do talhão e povoamento. Consideram-se como unidade primária o talhão e como unidade secundária à parcela quadrada de $400 \mathrm{~m} 2$. A tabela 2 apresenta a área, o número de parcelas lançadas aleatoriamente $\left(m_{i}\right)$ e as frações de subamostragem $\left(\frac{m_{i}}{M_{i}}\right)$ para os talhões selecionados do povoamento, bem como o total de parcelas lançadas aleatoriamente e a intensidade amostral. 
Ignácio, S. A.

Tabela 2: Área, número potencial de parcelas $\left(\mathrm{M}_{\mathrm{i}}\right)$, número de parcelas lançadas aleatoriamente $\left(\mathrm{m}_{\mathrm{i}}\right)$ e fração de amostragem $\left(\mathrm{m}_{\mathrm{i}} / \mathrm{M}_{\mathrm{i}}\right)$, segundo os talhões selecionados pertencentes ao povoamento de Mogi Guaçu plantio com semente

Table 2: Area, plot potential number $\left(M_{i}\right)$, number of plots randomly chosen $\left(m_{i}\right)$ and sub-sampling fraction $\left(m_{i} / M i\right)$, according to the selected Mogi Guaçu stand - seed planting

\begin{tabular}{|c|c|c|c|c|c|}
\hline N. ${ }^{o}$ de Ordem & N. ${ }^{o}$ do Talhão & $\begin{array}{l}\text { Área } \\
\text { (ha) }\end{array}$ & $M_{i}$ & $m_{i}$ & $m_{i} / M_{i}$ \\
\hline 1 & 1 & 50 & 1250 & 5 & 0,0040 \\
\hline 2 & 2 & 50 & 1250 & 5 & 0,0040 \\
\hline 3 & 3 & 38 & 950 & - & - \\
\hline 4 & 4 & 51 & 1275 & - & - \\
\hline 5 & 5 & 50 & 1250 & 5 & 0,0040 \\
\hline 6 & 6 & 48 & 1200 & 5 & 0,0042 \\
\hline 7 & 7 & 29 & 725 & - & - \\
\hline 8 & 8 & 23 & 575 & 4 & 0,0070 \\
\hline 9 & 9 & 25 & 625 & 4 & 0,0064 \\
\hline 10 & 10 & 29 & 725 & - & - \\
\hline 11 & 11 & 28 & 700 & - & - \\
\hline 12 & 12 & 25 & 625 & 4 & 0,0064 \\
\hline 13 & 13 & 25 & 625 & - & - \\
\hline 14 & 14 & 28 & 700 & 7 & 0,0100 \\
\hline 15 & 15 & 15 & 375 & - & - \\
\hline 16 & 17 & 29 & 725 & 4 & 0,0055 \\
\hline 17 & 18 & 11 & 275 & - & - \\
\hline 18 & 19 & 20 & 500 & 4 & 0,0080 \\
\hline 19 & 20 & 10 & 250 & 3 & 0,0120 \\
\hline 20 & 21 & 15 & 375 & - & - \\
\hline 21 & 22 & 22 & 550 & - & - \\
\hline 22 & 23 & 16 & 400 & 4 & 0,0100 \\
\hline 23 & 24 & 17 & 425 & 3 & 0,0071 \\
\hline 24 & 25 & 17 & 425 & - & - \\
\hline 25 & 26 & 9 & 225 & - & - \\
\hline 26 & 28 & 11 & 275 & - & - \\
\hline 27 & 40 & 17 & 425 & 3 & 0,0071 \\
\hline 28 & 52 & 24 & 600 & 4 & 0,0067 \\
\hline 29 & 53 & 51 & 1275 & - & - \\
\hline 30 & 56 & 20 & 500 & 4 & 0,0080 \\
\hline 31 & 57 & 18 & 450 & - & - \\
\hline 32 & 62 & 46 & 1150 & - & - \\
\hline 33 & 63 & 15 & 375 & 3 & 0,0080 \\
\hline 34 & 64 & 33 & 825 & - & - \\
\hline 35 & 65 & 15 & 375 & - & - \\
\hline 36 & 70 & 24 & 600 & - & - \\
\hline 37 & 73 & 19 & 475 & 4 & 0,0084 \\
\hline 38 & 74 & 13 & 325 & 3 & 0,0092 \\
\hline 39 & 75 & 32 & 800 & - & - \\
\hline 40 & 77 & 26 & 650 & - & - \\
\hline 41 & 79 & 20 & 500 & - & - \\
\hline 42 & 80 & 29 & 725 & 4 & 0,0055 \\
\hline 43 & 85 & 13 & 325 & 4 & 0,0123 \\
\hline 44 & 86 & 14 & 350 & 4 & 0,0114 \\
\hline TOTAL & - & 1120 & 28000 & 90 & 0,0032 \\
\hline
\end{tabular}

FONTE DOS DADOS BRUTOS: International Paper do Brasil Ltda.

De acordo com a tabela 2, a intensidade de amostragem para unidades primárias (talhões) foi de $0,5(50 \%)$, conforme sugerem Hansen et al. (1956) quando não se dispõe de informações para a determinação do número ótimo de unidades primárias. Para as 
unidades secundárias (parcelas), dentro das unidades primárias selecionadas, tomou-se o número de parcelas efetivamente amostradas pela empresa. Dessa forma, a intensidade média de amostragem por talhão selecionado foi de 0,0032 ou $0,32 \%$, o que equivale, em média, a uma parcela de 0,04 hectare $(400 \mathrm{~m} 2)$ amostrada para aproximadamente 12 hectares do povoamento.

Estimadores para a subamostragem com unidades primárias de tamanhos desiguais

Considerando uma amostragem em dois estágios, em uma população formada de $i^{\prime} s$ unidades primárias (talhões), contendo números variáveis de $j^{\prime} s$ unidades secundárias (parcelas quadradas) dentro das unidades primárias selecionadas, com $i=1,2, \ldots, N \mathrm{e}$ $j=1,2, \ldots, M_{i}$, e sendo $n$ o número de unidades primárias selecionadas com reposição e $m_{i} \mathrm{o}$ número de unidades secundárias amostradas na i-ésima unidade primária selecionada, são apresentados os estimadores para o i-ésimo talhão selecionado e para o povoamento.

Estimadores para o Talhão Selecionado

As fórmulas a seguir foram empregadas no cálculo das estimativas para o talhão selecionado:

a) Volume médio estimado de árvores $\left(\bar{v}_{i}\right)$, em $\mathrm{m}^{3} /$ ha, para o i-ésimo talhão selecionado

$\bar{v}_{i}=\frac{\sum_{j=1}^{m_{i}} v_{j}}{m_{i}}$

em que

$v_{j}=$ volume de árvores, $\mathrm{em} \mathrm{m}^{3} / \mathrm{ha}$, obtido na $\mathrm{i}$ ésima parcela amostrada; e

$m_{i}=$ número de parcelas amostradas no iésimo talhão selecionado.

b) Volume total estimado de árvores $\left(v t_{i}\right), \mathrm{em} \mathrm{m}^{3}$, para o iésimo talhão selecionado em que

$A_{i}=$ área em hectare do i-ésimo talhão selecionado.

c) Variância do volume médio estimado de árvores por hectare $\left(S_{\bar{v}_{i}}^{2}\right)$, para o i-ésimo talhão selecionado

$S_{\bar{v}_{i}}^{2}=\frac{S_{i}^{2}}{m_{i}}$

em que

$S_{i}^{2}=$ variância entre as parcelas amostradas dentro do i-ésimo talhão selecionado, sendo dada por:

$$
S_{i}^{2}=\frac{\sum_{j=1}^{m_{i}}\left(v_{j}-\bar{v}_{i}\right)^{2}}{m_{i}-1}=\frac{\sum_{j=1}^{m_{i}} v_{j}^{2}-m_{i} \cdot \bar{v}_{i}^{2}}{m_{i}-1}
$$

d) Erro padrão do volume médio estimado de árvores, $\left(S_{\bar{v}_{i}}\right)$, em $\mathrm{m}^{3} /$ ha, para o i-ésimo talhão selecionado

$$
S_{\bar{v}_{i}}=\sqrt{S_{\bar{v}_{i}}^{2}}
$$

e) Erro de amostragem absoluto $\left(E_{a}\right)$, em $\mathrm{m}^{3} / \mathrm{ha}$, para o iésimo talhão selecionado

$E_{a}= \pm t_{\left(m_{i}-1\right) ; \frac{\alpha}{2}} \cdot S_{\bar{v}_{i}}$

em que

$t=$ valor tabelado da estatística t de Student;

$\alpha=$ nível de significância; e

$m_{i}-1=$ número de graus de liberdade da amostra de unidades secundárias (parcelas) amostradas no i-ésimo talhão selecionado.

f) Erro de amostragem relativo $\left(E_{\%}\right)$, para o i-ésimo talhão selecionado

$v t_{i}=\bar{v}_{i} \cdot A_{i}$ 
$E_{\%}=\left( \pm \frac{t_{\left(m_{i}-1\right) ; \frac{\alpha}{2}} \cdot s_{\bar{v}_{i}}}{\bar{v}_{i}}\right) 100$

g) Intervalo de confiança para o volume médio verdadeiro de árvores $\left(\bar{V}_{i}\right), \mathrm{em} \mathrm{m}^{3} /$ ha, para o i-ésimo talhão selecionado

$P\left\{\bar{v}_{i}-t . S_{\bar{v}_{i}} \leq \bar{V}_{i} \leq \bar{v}_{i}+t . S_{\bar{v}_{i}}\right\}=(1-\alpha)$

h) Intervalo de confiança para o volume total verdadeiro de árvores $\left(V T_{i}\right)$, em $\mathrm{m}^{3}$, para o iésimo talhão selecionado

$P\left\{v t_{i}-A . t . S_{\bar{v}_{i}} \leq V T_{i} \leq v t_{i}+\right.$ A.t. $\left.S_{\bar{v}_{i}}\right\}=(1-\alpha)$

\section{Estimadores para o povoamento}

As fórmulas a seguir foram empregadas no cálculo das estimativas para o povoamento, considerando os dois processos de subamostragem com unidades primárias de tamanhos desiguais estudados nesta pesquisa.

\section{Processo I:}

Unidades primárias selecionadas com probabilidades iguais - estimador tendencioso por índice em relação ao tamanho

a) Volume médio estimado de árvores $\left(\overline{\bar{v}}_{I}\right)$, em $\mathrm{m}^{3} / \mathrm{ha}$

$$
\overline{\bar{v}}_{I}=\frac{\sum_{i=1}^{n} M_{i} \bar{v}_{i}}{\sum_{i=1}^{n} M_{i}}
$$

em que

$\overline{\bar{v}}_{I}=$ volume médio estimado de árvores, em $\mathrm{m}^{3} / \mathrm{ha}$, para o povoamento, obtido com o processo I;

$\bar{v}_{i}=$ volume médio estimado de árvores, em $\mathrm{m}^{3} /$ ha, para o i-ésimo talhão selecionado;
$M_{i}=$ número de parcelas potenciais no iésimo talhão selecionado;

b) Volume total estimado $\left(v t_{I}\right)$, $\mathrm{em} \mathrm{m}^{3}$

$v t_{I}=\overline{\bar{v}}_{I} \cdot A$

em que

$A=$ área do povoamento em hectare.

c) Variância do volume médio estimado de árvores por hectare $\left(S_{\overline{\bar{v}}_{I}}^{2}\right)$

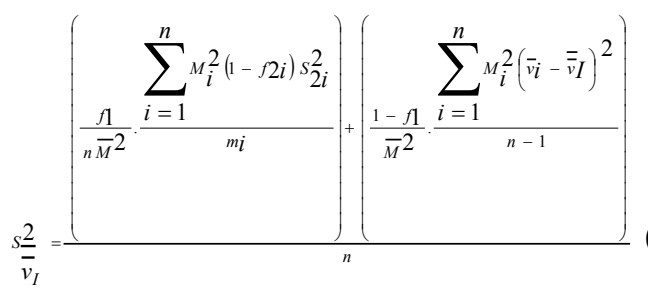

em que

$S_{\overline{\bar{v}}_{I}}^{2}=$ variância do volume médio, obtida com o processo I,

$\overline{\bar{v}}_{I}=$ volume médio estimado de árvores, em $\mathrm{m}^{3} /$ ha, para o povoamento, obtido com o processo I;

$\bar{v}_{i}=$ volume médio estimado de árvores, em $\mathrm{m}^{3} /$ ha, para o i-ésimo talhão selecionado;

$n=$ número de talhões selecionados;

$N=$ número de talhões do povoamento;

$m_{i}=$ número de parcelas amostradas no iésimo talhão selecionado;

$M_{i}=$ número de parcelas potenciais no i-ésimo talhão selecionado;

$M_{0}=\sum_{i=1}^{n} M_{i}=$ número total de parcelas potenciais dos talhões selecionados;

$f_{1}=\frac{n}{N}=$ fração de amostragem de unidades primárias (talhões);

$f_{2 i}=\frac{m_{i}}{M_{i}}=$ fração de amostragem de unidades secundárias (parcelas) do i-ésimo talhão selecionado;

$\bar{M}=$ número médio de parcelas potenciais por talhão selecionado, sendo dado por: 
$\bar{M}=\frac{\sum_{i=1}^{n} M_{i}}{n}=\frac{M_{0}}{n}$

$S_{2 i}^{2}=$ = variância entre as parcelas amostradas dentro do i-ésimo talhão selecionado, sendo dada por:

$s_{2 i}^{2}=\frac{1}{m_{i}-1} \sum_{j=1}^{m_{i}}\left(v_{i j}-\bar{v}_{i}\right)^{2}$

em que

$v_{i j}=$ volume de árvores da j-ésima parcela, no i-ésimo talhão selecionado.

d) Erro padrão do volume médio estimado de árvores $\left(S_{\overline{\bar{v}}_{I}}\right)$, em $\mathrm{m}^{3} / \mathrm{ha}$

$S_{\overline{\bar{v}}_{I}}=\sqrt{S_{\overline{\bar{v}}_{I}}^{2}}$

e) Erro de amostragem absoluto $\left(E_{a}\right), \mathrm{em} \mathrm{m}^{3} / \mathrm{ha}$

$E_{a}= \pm t_{(n-1) ; \frac{\alpha}{2}} \cdot S_{\overline{\bar{v}}_{I}}$

em que

$t=$ valor tabelado da estatística t de Student;

$\alpha=$ nível de significância; e

$n-1=$ número de graus de liberdade da amostra de unidades primárias (talhões).

f) Erro de amostragem relativo $\left(E_{\%}\right)$

$E_{\%}=\left( \pm \frac{t_{(n-1) ; \frac{\alpha}{2}} \cdot S_{\overline{\bar{v}}_{I}}}{\overline{\bar{v}}_{I}}\right) 100$

g) Intervalo de confiança para o volume médio verdadeiro de árvores $\left(\overline{\bar{V}}_{I}\right), \mathrm{em} \mathrm{m} \mathrm{m}^{3} / \mathrm{ha}$

$P\left\{\overline{\bar{v}}_{I}-t . S_{\overline{\bar{v}}_{I}} \leq \overline{\bar{V}}_{I} \leq \overline{\bar{v}}_{I}+t . S_{\overline{\bar{v}}_{I}}\right\}=(1-\alpha)$

h) Intervalo de confiança para o volume total verdadeiro de árvores $\left(V T_{I}\right), \mathrm{em} \mathrm{m}^{3}$
$P\left\{v t_{I}-\right.$ A.t. $S_{\overline{\bar{v}}_{I}} \leq V T_{I} \leq v t_{I}+$ A.t. $\left.S_{\overline{\bar{v}}_{I}}\right\}=(1-\alpha)$

\section{Processo II:}

Unidades primárias selecionadas com probabilidade proporcional ao tamanho do talhão - estimador não tendencioso

a) Volume médio estimado de árvores $\left(\overline{\bar{v}}_{I I}\right), \mathrm{em} \mathrm{m}^{3} / \mathrm{ha}$

$\overline{\bar{v}}_{I I}=\frac{\sum_{i=1}^{n} \bar{v}_{i}}{n}$

em que

$\overline{\bar{v}}_{I I}=$ volume médio estimado de árvores, em $\mathrm{m}^{3} /$ ha, para o povoamento, obtido com o processo II;

$n=$ número de talhões selecionados; e

$\bar{v}_{i}=$ volume médio estimado de árvores, em $\mathrm{m}^{3} /$ ha, para o i-ésimo talhão selecionado.

b) Volume total estimado $v t_{\| l}$, $\mathrm{em} \mathrm{m}^{3}$

$v t_{I I}=\overline{\bar{v}}_{I I} \cdot A$

em que

$A=$ a área do povoamento em hectare.

c) Variância do volume médio estimado de árvores por hectare $\left(S_{\overline{\bar{v}}_{I i}}^{2}\right)$

$S_{\overline{\bar{v}}_{I I}}^{2}=\frac{\frac{1}{(n-1)} \sum_{i=1}^{n}\left(\bar{v}_{i}-\overline{\bar{v}}_{I I}\right)^{2}}{n}$

em que

$S_{\overline{\bar{V}}_{I I}}^{2}=$ variância do volume médio, obtida com o processo II,

$\overline{\bar{v}}_{I I}=$ volume médio estimado de árvores, em $\mathrm{m}^{3} / \mathrm{ha}$, para o povoamento, obtido com o processo II;

$\bar{v}_{i}=$ volume médio estimado de árvores, em $\mathrm{m}^{3} /$ ha, para o i-ésimo talhão selecionado; e 
$n=$ número de talhões selecionados.

d) Erro padrão do volume médio estimado de árvores $\left(S_{\overline{\bar{v}}_{I I}}\right)$, em $\mathrm{m}^{3} / \mathrm{ha}$

$S_{\overline{\bar{v}}_{I I}}=\sqrt{S_{\overline{\bar{v}}_{I I}}^{2}}$

e) Erro de amostragem absoluto $\left(E_{a}\right), \mathrm{em} \mathrm{m}^{3} / \mathrm{ha}$

$E_{a}= \pm t_{(n-1) ; \frac{\alpha}{2}} \cdot S_{\overline{\bar{v}}_{I I}}$

em que

$t=$ valor tabelado da estatística $t$ de Student;

$\alpha=$ nível de significância; e

$n-1=$ número de graus de liberdade da amostra de unidades primárias (talhões).

f) Erro de amostragem relativo $\left(E_{\%}\right)$

$E_{\%}=\left( \pm \frac{t_{(n-1) ; \frac{\alpha}{2}} \cdot S_{\overline{\bar{v}}_{I I}}}{\overline{\bar{v}}_{I I}}\right) 100$

g) Intervalo de confiança para o volume médio verdadeiro de árvores $\left(\overline{\bar{V}}_{I I}\right), \mathrm{em} \mathrm{m}^{3} / \mathrm{ha}$

$P\left\{\overline{\bar{v}}_{I I}-t . S_{\overline{\bar{v}}_{I I}} \leq \overline{\bar{V}}_{I I} \leq \overline{\bar{v}}_{I I}+t . S_{\overline{\bar{v}}_{I I}}\right\}=(1-\alpha)$

h) Intervalo de confiança para o volume total verdadeiro de árvores $\left(V T_{I I}\right), \mathrm{em} \mathrm{m}^{3}$

$P\left\{v t_{I I}-\right.$ A.t. $S_{\overline{\bar{v}}_{I I}} \leq V T_{I I} \leq v t_{I I}+$ A.t. $\left.S_{\overline{\bar{v}}_{I I}}\right\}=(1-\alpha)$

\section{CÁlCULO DA ESTRUTURA DE PESO $Z_{i}$}

Para a definição do peso da unidade primária (talhão), em que o sorteio é feito com probabilidade proporcional à área do i-ésimo talhão, a variável $Z_{i}$ foi obtida a partir do quociente entre o tamanho do i-ésimo talhão selecionado e a área total dos talhões selecionados na amostra, ou seja:

$Z_{i}=\frac{M_{i}}{M_{0}}=\frac{A_{i}}{A}$

em que

$Z_{i}=$ proporção referente ao tamanho do iésimo talhão selecionado em relação ao valor total do tamanho dos talhões selecionados na amostra;

$M_{i}=$ número de parcelas potenciais no i-ésimo talhão selecionado;

$M_{0}=\sum_{i=1}^{n} M_{i}=$ número total de parcelas potenciais dos talhões selecionados;

$A_{i}=$ área do i-ésimo talhão selecionado; e

$A=\sum_{i=1}^{n} A_{i}=$ área total dos talhões selecionados.

A operacionalização do sorteio das unidades primárias com probabilidade proporcional ao tamanho conhecido do i-ésimo talhão, é feita organizando-se uma listagem das unidades primárias com seus respectivos tamanhos expressos por $M_{i}$ ou $A_{i}$.

Tomando-se o somatório cumulativo do tamanho do talhão expresso por $M_{i}$ ou $A_{i}$, são construídos os intervalos para cada unidade primária, cuja gama de variação permitirá, por meio de um sorteio aleatório, receber uma alocação distributiva segundo o critério com probabilidade proporcional ao tamanho do talhão. Para o sorteio das unidades primárias, pode-se usar uma tabela ou um programa gerador de números aleatórios, em que as combinações de dígitos seriam utilizadas dentro do intervalo total da gama de variação para sortear a i-ésima unidade primária cujo número aleatório sorteado caísse dentro do intervalo de variação do somatório acumulado do tamanho do talhão expresso por $M_{i}$ ou $A_{i}$.

Para selecionar uma segunda unidade primária, repete-se o processo com um novo número aleatório entre 1 e o somatório total do tamanho dos talhões, sem impedir o sorteio da unidade primária já selecionada. As 
unidades primárias são selecionadas com probabilidade proporcional ao tamanho do talhão e com reposição. Se a i-ésima unidade primária for selecionada mais de uma vez, toda a subamostra é reposta e faz-se um novo sorteio independente de $m_{i}$ subunidades, sem reposição.

\section{Comparação dos processos de subamostragem com unidades primárias de tamanhos desiguais}

Os critérios utilizados para a comparação entre as estimativas obtidas pelos dois processos de subamostragem, que empregam parcelas de área fixa com diferentes probabilidades de seleção das unidades primárias (talhões), foram: a) Eficiência Relativa (ER), conforme proposto por Yamane (1967), que utiliza a razão entre as variâncias dos volumes médios obtidos pelos dois processos de amostragem; b) eficiência relativa (ER), conforme proposto por Husch et al. (1982), que utiliza a razão entre os erros de amostragem relativos e respectivos tempos médios de medição para cada processo utilizado e c) análise de regressão, visando verificar o grau de relacionamento entre a variância do volume total do i-ésimo talhão selecionado $\left(S_{V_{i}}^{2}\right)$ e o seu tamanho $\left(M_{i}\right)$.

Segundo Cochran (1953), a variância do volume total $\left(S_{V_{i}}^{2}\right)$ do i-ésimo talhão selecionado é função do tamanho $\left(M_{i}\right)$ do respectivo talhão. Expressando essa relação em termos de função, tem-se:

$S_{V_{i}}^{2}=f\left(M_{i}\right)$

em que

$S_{V_{i}}^{2}=$ variância do volume total de árvores do i-ésimo talhão selecionado;

$f=$ relação funcional; e

$M_{i}=$ número de unidades secundárias potenciais (parcelas) no i-ésimo talhão selecionado.

Segundo Cochran (1953), em alguns tipos de levantamentos como, por exemplo, a amostragem de tipos de solo, a colheita de safra de cereais ou levantamentos agrários, que utilizem áreas como unidades amostrais, o tamanho da unidade primária pode-se apresentar com uma variação quase contínua, de forma que é de se esperar que $S_{V_{i}}^{2}$ aumente à medida que cresça o tamanho da unidade primária.

Segundo o autor, em vários levantamentos agrários, $S_{V_{i}}^{2}$ se apresenta correlacionada com $M_{i}$ pela fórmula empírica:

$S_{V_{i}}^{2}=\beta_{0} M_{i}^{\beta_{1}}$

em que

$\beta_{0}=$ coeficiente linear; e

$\beta_{1}=$ coeficiente angular.

Segundo a fórmula (37), expressa pelo modelo geométrico, $S_{V_{i}}^{2}$ cresce à medida que $M_{i}$ aumenta. É de se esperar uma curva da forma apresentada acima, quando há forças que exercem influência semelhante sobre elementos muito aproximados. As condições climáticas, os diferentes tipos de solo e a topografia tendem a produzir aspectos agrários semelhantes e aproximados à medida que $M_{i}$ diminuiu.

No presente trabalho, a forma funcional adotada para a estimativa da função (37) foi a linear no logaritmo decimal da variável dependente e independente, uma vez que este tipo de função apresenta a vantagem de se tornar linear quando sujeita à transformação logarítmica.

Com relação aos coeficientes, estabeleceu-se que:

a) $\quad \beta_{0}=0$, considerando que, para $M_{i}=0$, a variância do volume de árvores $S_{V_{i}}^{2}=0$, uma vez que não existe talhão;

b) $\quad \beta_{1}>0$, considerando que as variações no tamanho do iésimo talhão selecionado $\left(M_{i}\right)$ induzam a variações, no mesmo sentido, na variância do volume total de árvores $S_{V_{i}}^{2}$.

As hipóteses referentes aos coeficientes parciais de regressão das equações ajustadas, para os cinco povoamentos nas duas 
medições, foram testadas por meio do teste $t$ de student, enquanto o grau de ajustamento da regressão foi indicado pelo coeficiente de determinação $R^{2}$ e pelo coeficiente de determinação ajustado $\bar{R}^{2}$.

Utilizou-se, ainda, o teste $\mathrm{F}$ de Snedecor para testar a hipótese segundo a qual a variável independente "tamanho do i-ésimo talhão selecionado $\left(M_{i}\right)^{\prime}$ é estatisticamente relevante para explicar as variações na variância do volume total de árvores $\left(S_{V_{i}}^{2}\right)$ (Kmenta, 1978).

\section{RESULTADOS E DISCUSSÃO}

\section{Comparação dos processos de subamostragem com unidades primárias de tamanhos desiguais}

A variável tempo $\mathrm{T}_{\mathrm{i}}$, empregada nos cálculos da eficiência relativa do i-ésimo processo de subamostragem, não foi considerada devido à falta de dados, sendo portanto mantida constante para os dois processos. Todavia, pode-se considerar que o tempo médio gasto na alocação e medição das parcelas de área fixa é o mesmo, uma vez que são parcelas de igual tamanho.

As estimativas obtidas para 0 volume de árvores por hectare, com base nos dois processos de subamostragem com unidades primárias de tamanhos desiguais, foram obtidas com o mesmo tamanho de amostra e estão apresentadas na tabela 3 . As tabelas 4 e 5 apresentam detalhadamente o processo de cálculo para a obtenção dessas estatísticas.

\section{Eficiência relativa}

Tabela 3: Comparação da precisão entre os processos de subamostragem com unidades primárias de tamanhos desiguais para a estimativa do volume médio de árvores por hectare, para o povoamento de Mogi Guaçu, em regime de alto fuste - plantio com semente

Table 3: Comparison of precision between sub-sampling processes of unequal size primary units to estimate the tree average volume per hectare in the Mogi Guaçu stand, in clear-cut regimes-seed planting

\begin{tabular}{|c|c|c|c|c|c|c|}
\hline Processo & $\begin{array}{c}\text { Probabilidade na Seleção } \\
\text { das Unidades Primárias } \\
\text { (Talhões) }\end{array}$ & $\begin{array}{c}\text { Situação } \\
\text { Quanto } \\
\text { à Tendência }\end{array}$ & $\overline{\bar{V}}$ & $S_{\overline{\bar{V}}}^{2}$ & $S_{\overline{\bar{v}}}$ & $\mathrm{E}_{\%}(0,05)$ \\
\hline I & Igual & $\begin{array}{l}\text { Com } \\
\text { tendência }\end{array}$ & 286,23131 & 71,71182 & 8,46828 & 6,15 \\
\hline II & Proporcional à Área do Talhão & $\begin{array}{l}\text { Sem } \\
\text { tendência }\end{array}$ & 294,57963 & 131,78748 & 11,47987 & 8,11 \\
\hline
\end{tabular}

FONTE DOS DADOS BRUTOS: International Paper do Brasil Ltda.

NOTAS:

1 Número de unidades primárias (talhões) no povoamento: $\mathrm{N}=44$.

2 Número de unidades primárias (talhões) selecionadas: $\mathrm{n}=22$.

3 Número de unidades secundárias (parcelas de $20 \times 20$ ) potenciais nas unidades primárias no povoamento: $\mathrm{M}_{0}=$ 28000.

4 Número de unidades secundárias lançadas aleatoriamente nas unidades primárias selecionadas $\mathrm{m}_{\mathrm{o}}=90$.

5 Intensidade amostral média para o povoamento $\left(\frac{m_{0}}{M_{0}}\right) \times 100=0,32 \%$.

6 Valor da estatística “t” para 95\% de confiança e $(\mathrm{n}-1=21)$ graus de liberdade $=2,08$. 
Subamostragem com unidades...

Tabela 4:-Estimativas para o processo de subamostragem I, com probabilidades iguais, da variável volume de árvores para o povoamento de Mogi Guaçu - (plantio com semente) - idade entre 6 e 8 anos

Table 4: $\quad$ Sub-sampling process I estimation, with equal probability of tree volume variable in the Mogi Guaçu stand - seed planting - age between 6 and 8 years

\begin{tabular}{|c|c|c|c|c|c|c|c|c|c|}
\hline $\begin{array}{l}\text { Talhão } \\
\text { Sorteado }\end{array}$ & $\begin{array}{c}\text { Parcela } \\
400 \mathrm{~m}^{2} \\
\left(M_{i}\right)\end{array}$ & $\begin{array}{l}\text { Área } \\
\text { (ha) }\end{array}$ & $\begin{array}{c}\text { Parcela } \\
400 \mathrm{~m}^{2} \\
\left(m_{i}\right)\end{array}$ & $\begin{array}{c}\text { Volume } \\
\text { Parcela } \\
400 \mathrm{~m}^{2}\left(v_{i}\right)\end{array}$ & $\bar{v}_{i}$ & $M_{i} \bar{v}_{i}$ & $S_{2 i}^{2}$ & $\left(M_{i}^{2}\left(\left(1-f_{2 i}^{2}\right)\right) / m_{i}\right.$ & $M_{i}^{2}\left(\bar{v}_{i}-\overline{\bar{v}}_{I}\right)^{2}$ \\
\hline 1 & 1250 & 50 & 5 & 47,52 & 9,50 & 11880,00000 & 0,63914 & 198932,97863 & 5912520,83564 \\
\hline 2 & 1250 & 50 & 5 & 62,35 & 12,47 & 15587,50000 & 0,75099 & 233747,78512 & 1628003,57213 \\
\hline 5 & 1250 & 50 & 5 & 57,74 & 11,55 & 14435,00000 & 2,16413 & 673585,15125 & 15235,56538 \\
\hline 6 & 1200 & 48 & 5 & 46,51 & 9,30 & 11162,40000 & 1,13903 & 326673,80400 & 6639407,96062 \\
\hline 8 & 575 & 23 & 4 & 37,37 & 9,34 & 5371,93750 & 0,26047 & 21379,39128 & 1467450,18051 \\
\hline 9 & 625 & 25 & 4 & 33,20 & 8,30 & 5187,50000 & 1,99127 & 193215,12609 & 3874141,05351 \\
\hline 12 & 625 & 25 & 4 & 37,95 & 9,49 & 5929,68750 & 2,81068 & 272723,40563 & 1503312,09711 \\
\hline 14 & 700 & 28 & 7 & 71,81 & 10,26 & 7181,00000 & 6,77280 & 469354,90140 & 694685,30617 \\
\hline 17 & 725 & 29 & 4 & 43,86 & 10,97 & 7949,62500 & 1,60094 & 209213,62446 & 123260,10790 \\
\hline 19 & 500 & 20 & 4 & 40,90 & 10,23 & 5112,50000 & 0,11315 & 7015,52940 & 374699,49722 \\
\hline 20 & 250 & 10 & 3 & 29,48 & 9,83 & 2456,66666 & 4,56843 & 94033,64100 & 164549,36436 \\
\hline 23 & 400 & 16 & 4 & 44,36 & 11,09 & 4436,00000 & 3,22147 & 127570,17240 & 20650,15610 \\
\hline 24 & 425 & 17 & 3 & 47,53 & 15,84 & 6733,41667 & 2,53966 & 151829,34033 & 3487495,34621 \\
\hline 40 & 425 & 17 & 3 & 40,71 & 13,57 & 5767,25000 & 0,77253 & 46184,45437 & 812372,38321 \\
\hline 52 & 600 & 24 & 4 & 60,54 & 15,14 & 9081,00000 & 9,90736 & 885717,62640 & 4890500,34410 \\
\hline 56 & 500 & 20 & 4 & 53,08 & 13,27 & 6635,00000 & 2,23441 & 138533,23400 & 828778,94992 \\
\hline 63 & 375 & 15 & 3 & 38,50 & 12,83 & 4812,50000 & 1,43123 & 66552,38100 & 269391,86034 \\
\hline 73 & 475 & 19 & 4 & 58,52 & 14,63 & 6949,25000 & 5,14722 & 287890,61641 & 2282680,78933 \\
\hline 74 & 325 & 13 & 3 & 42,23 & 14,08 & 4574,91667 & 0,24742 & 8630,71573 & 729160,74952 \\
\hline 80 & 725 & 29 & 4 & 50,68 & 12,67 & 9185,75000 & 5,34900 & 699013,61421 & 783297,23680 \\
\hline 85 & 325 & 13 & 4 & 44,70 & 11,18 & 3631,87500 & 2,07525 & 54125,00974 & 7944,61460 \\
\hline 86 & 350 & 14 & 4 & 54,83 & 13,71 & 4797,62500 & 3,62020 & 109601,52472 & 624710,15717 \\
\hline TOTAL & 13875 & 555 & 90 & 1044,37 & 259,23 & 158858,40000 & - & 5275524,02758 & 37134248,12780 \\
\hline
\end{tabular}

FONTE DOS DADOS BRUTOS: International Paper do Brasil Ltda.

NOTA: $\mathrm{N}=44 ; \mathrm{n}=22$

Média global viesada $\overline{\bar{v}}_{l}=$

Variação dentro =

Variação entre $=$

Variância da média $=$

Erro padrão da média $=$

Valor da estatística " $\mathrm{t} "=$

Erro de amostragem $(0,05)=$

Erro relativo $(\%)=$

\section{PARCELA}

11,44925

0,01370

0,10104

0,11474

0,33873

2,08

0,70456

6,15
HECTARE

286,23135

8,56347

63,14836

71,71182

8,46828

2,08

17,61403

6,15 
Tabela 5: Estimativas para o processo de subamostragem II, com probabilidade proporcional à área do talhão, da variável volume de árvores para o povoamento de Mogi Guaçu - plantio com semente - idade entre 6 e 8 anos

Table 5: $\quad$ Sub-sampling II process estimation, with probability proportional to stand area, tree volume variable in the Mogi Guaçu stand - seed planting - age between 6 and 8 years

\begin{tabular}{|c|c|c|c|c|c|c|c|c|}
\hline $\begin{array}{l}\text { Talhão } \\
\text { Sorteado }\end{array}$ & $\begin{array}{c}\text { Parcela } \\
400 \mathrm{~m}^{2}\left(M_{i}\right)\end{array}$ & $\begin{array}{l}\text { Área } \\
\text { (ha) }\end{array}$ & $Z_{i}=M_{i} / \lambda$ & $\begin{array}{c}\text { Parcela } \\
400 \mathrm{~m}^{2} \\
\left(m_{i}\right)\end{array}$ & $\begin{array}{c}\text { Volume } \\
\text { Parcela } \\
400 \mathrm{~m}^{2}\left(v_{i}\right)\end{array}$ & $\bar{v}_{i}$ & $\left(\bar{v}_{i}-\overline{\bar{v}}_{I I}\right)^{2}$ & $S_{2 i}^{2}$ \\
\hline 1 & 1250 & 50 & 0,09009 & 5 & 47,52 & 9,50 & 5,19469 & 0,63914 \\
\hline 2 & 1250 & 50 & 0,09009 & 5 & 62,35 & 12,47 & 0,47172 & 0,75099 \\
\hline 5 & 1250 & 50 & 0,09009 & 5 & 57,74 & 11,55 & 0,05531 & 2,16413 \\
\hline 6 & 1200 & 48 & 0,08649 & 5 & 46,51 & 9,30 & 6,15628 & 1,13903 \\
\hline 8 & 575 & 23 & 0,04144 & 4 & 37,37 & 9,34 & 5,95694 & 0,26047 \\
\hline 9 & 625 & 25 & 0,04504 & 4 & 33,20 & 8,30 & 12,13258 & 1,99127 \\
\hline 12 & 625 & 25 & 0,04504 & 4 & 37,95 & 9,49 & 5,27017 & 2,81068 \\
\hline 14 & 700 & 28 & 0,05045 & 7 & 71,81 & 10,26 & 2,32444 & 6,77280 \\
\hline 17 & 725 & 29 & 0,05225 & 4 & 43,86 & 10,97 & 0,66943 & 1,60095 \\
\hline 19 & 500 & 20 & 0,03604 & 4 & 40,90 & 10,23 & 2,42794 & 0,11315 \\
\hline 20 & 250 & 10 & 0,01802 & 3 & 29,48 & 9,83 & 3,82796 & 4,56844 \\
\hline 23 & 400 & 16 & 0,02883 & 4 & 44,36 & 11,09 & 0,48051 & 3,22147 \\
\hline 24 & 425 & 17 & 0,03063 & 3 & 47,53 & 15,84 & 16,48480 & 2,53966 \\
\hline 40 & 425 & 17 & 0,03063 & 3 & 40,71 & 13,57 & 3,19271 & 0,77253 \\
\hline 52 & 600 & 24 & 0,04324 & 4 & 60,54 & 15,14 & 11,23466 & 9,90736 \\
\hline 56 & 500 & 20 & 0,03604 & 4 & 53,08 & 13,27 & 2,21062 & 2,23441 \\
\hline 63 & 375 & 15 & 0,02703 & 3 & 38,50 & 12,83 & 1,10281 & 1,43123 \\
\hline 73 & 475 & 19 & 0,03423 & 4 & 58,52 & 14,63 & 8,10440 & 5,14722 \\
\hline 74 & 325 & 13 & 0,02342 & 3 & 42,23 & 14,08 & 5,26006 & 0,24742 \\
\hline 80 & 725 & 29 & 0,05225 & 4 & 50,68 & 12,67 & 0,78644 & 5,34899 \\
\hline 85 & 325 & 13 & 0,02342 & 4 & 44,70 & 11,18 & 0,36989 & 2,07525 \\
\hline 86 & 350 & 14 & 0,02522 & 4 & 54,83 & 13,71 & 3,70299 & 3,62020 \\
\hline TOTAL & 13875 & 555 & 1,00000 & 90 & 1044,37 & 259,23 & 97,41730 & 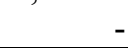 \\
\hline
\end{tabular}

FONTE DOS DADOS BRUTOS: International Paper do Brasil Ltda. NOTA: $\mathrm{N}=44 ; \mathrm{n}=22$

$\begin{array}{lrr} & \text { PARCELA } & \text { HECTARE } \\ \text { Média global não viesada } \overline{\bar{v}}_{\|}= & 11,78319 & 294,57963 \\ \text { Variância da média }= & 0,21086 & 131,78748 \\ \text { Erro padrão da média }= & 0,45919 & 11,47987 \\ \text { Valor da estatística "t" }= & 2,08 & 2,08 \\ \text { Erro de amostragem }(0,05)= & 0,95513 & 23,87814 \\ \text { Erro relativo (\%) }= & 8,11 & 8,11\end{array}$

Comparando a estimativa do volume de árvores por hectare obtidas pelo processo I com a estimativa obtida pelo processo II, chegou-se a resultados próximos para os volumes médios, com uma variação de $2,92 \%$, obtendo-se estimativas de $286,23 \mathrm{~m}^{3} /$ ha pelo processo I e de $294,58 \mathrm{~m}^{3} /$ ha pelo processo II. Com relação à precisão relativa, constata-se que ocorre maior heterogeneidade, medida pelo erro de amostragem relativo, no processo II. A eficiência relativa do processo I em relação ao processo II é de $75,83 \%$, implicando na obtenção de intervalos de confiança mais estreitos para a média e total.
Análise de regressão

A partir do modelo previamente estabelecido, estimou-se, por meio do método de mínimos quadrados ordinários, a equação (37) para o povoamento analisado. O coeficiente linear $\beta_{0}$ somente foi estatisticamente significante a um nível de probabilidade maior que $50 \%$. Dessa forma, procedeu-se ao ajuste do modelo excluindo o intercepto. As tabelas 6 e 7 apresentam as estimativas com e sem o coeficiente linear $\beta_{0}$, o valor do coeficiente de determinação $R^{2}$, do 
coeficiente de determinação ajustado $\bar{R}^{2}$ e a estatística F.

Com relação ao poder explicativo dos determinação $R^{2}, \bar{R}^{2}$ e $\mathrm{F}$, os três valores aumentaram com a exclusão do intercepto e indicam alto poder explicativo. modelos, indicado pelo coeficiente de

Tabela 6: Estimativa da equação da variância do volume total de árvores com o intercepto, em nível de talhão, para o povoamento de Mogi Guaçu, em regime de alto fuste - plantio com semente

Table 6: Estimation of the equation of stand tree total volume with linear coefficient in the Mogi Guaçu stand, in clear-cut regimes- seed planting

\begin{tabular}{lr|r|r|r}
\hline \multicolumn{1}{c|}{ Variáveis } & \multicolumn{1}{c|}{$\begin{array}{c}\text { Coeficientes } \\
\text { Estimados }\end{array}$} & $\begin{array}{c}\text { Erros } \\
\text { Padrões }\end{array}$ & $\begin{array}{c}\text { Estatística } \\
\mathrm{t}\end{array}$ & \multicolumn{2}{c}{ Significância } \\
\hline LOG $\beta_{O}$ & 0,80221 & 1,48192 & 0,54133 & 0,59426 \\
LOG $M_{I}$ & 1,79099 & 0,53712 & 3,33444 & 0,00330 \\
$R^{2}$ & 0,35730 & - & - & - \\
$\bar{R}^{2}$ & 0,32516 & - & - & - \\
$F$ & 11,11852 & - & - & 0,00330 \\
\hline
\end{tabular}

FONTE DOS DADOS BRUTOS: International Paper do Brasil Ltda.

NOTA: As variáveis dependente e independente estão expressas nos logaritmos decimais dos valores observados.

Tabela 7: Estimativa da equação da variância do volume total de árvores sem o intercepto, em nível de talhão, para o povoamento de Mogi Guaçu, em regime de alto fuste - plantio com semente

Table 7: Estimation of the equation of stand tree total volume without linear coefficient in the Mogi Guaçu stand, in clear-cut regimes- seed planting

\begin{tabular}{l|r|r|r|r}
\hline \multicolumn{1}{c|}{ Variáveis } & \multicolumn{1}{c|}{$\begin{array}{c}\text { Coeficientes } \\
\text { Estimados }\end{array}$} & $\begin{array}{c}\text { Erros } \\
\text { Padrões }\end{array}$ & $\begin{array}{c}\text { Estatística } \\
\text { t }\end{array}$ & \multicolumn{2}{c}{ Significância } \\
\hline LOG $M_{I}$ & 2,08099 & 0,03829 & 54,35183 & 0,00000 \\
$R^{2}$ & 0,99294 & - & - & - \\
$\bar{R}^{2}$ & 0,99261 & - & - & - \\
$F$ & 2954,12200 & - & - & 0,00000 \\
\hline
\end{tabular}

FONTE DOS DADOS BRUTOS: International Paper do Brasil Ltda.

NOTA: As variáveis dependente e independente estão expressas nos logaritmos decimais dos valores observados.

Após a exclusão do intercepto, o coeficiente $\beta_{1}$ mostrou-se estatisticamente significante a um nível de probabilidade menor que $1 \%$, sendo maior que 1 .

Os resultados obtidos confirmaram o estabelecido em Cochran (1953), ou seja, quando $\beta_{1}$ é maior que 1 , as estimativas por índice, em que as unidades primárias são selecionadas com probabilidades iguais (Processo I), são mais precisas que as estimativas para unidades primárias selecionadas com probabilidades proporcionais ao tamanho (Processo II).

\section{CONCLUSÕES}

Com base na análise dos resultados, as principais conclusões deste trabalho são:
- $\mathrm{O}$ erro de amostragem relativo do processo de subamostragem I, por índice, em que as unidades primárias são selecionadas com probabilidades iguais, foi inferior ao processo de subamostragem II, em que as unidades primárias são selecionadas com probabilidades proporcionais à área do talhão, confirmando as pressuposições estabelecidas em Cochran (1953), ou seja, quando no ajuste do modelo da variância total do i-ésimo talhão selecionado em função do tamanho $M_{i}$ a equação apresentar $\beta_{1}$ maior que 1 , as estimativas por índice, com probabilidades iguais (processo I), são mais precisas que as estimativas para as unidades primárias selecionadas com probabilidades proporcionais ao tamanho (processo II);

- A adoção de processos de subamostragem com unidades primárias de tamanhos desiguais, utilizando uma intensidade de amostragem igual a $50 \%$ de $\mathrm{N}$, em média, é muito eficiente 
para uso nos inventários contínuos de florestas plantadas.

- Os custos dos inventários (medição de parcelas dentro dos talhões selecionados) realizados por processos de subamostragem com unidades primárias de tamanhos desiguais, podem ser, significativamente reduzidos e apresentar uma precisão admissível, tanto mais quanto maior for a homogeneidade do povoamento, se o objetivo for obter informações seguras dos valores médios e totais da variável de interesse.

\section{REFERENCIAS}

COCHRAN, W. G. Sampling techniques. 3 ed. New York: J. Wiley e Sons, 1953. 422p.

HANSEN, M. H.; HURWITZ, W. N. On the theory of sampling from finite populations. Annals of Mathematical Statistics, Ann Arbor, v. 14, p. 333-362, 1943.

HANSEN, M. H.; HURWITZ, W. N.; MADOW, W. G. Sample survey methods and theory. 2.ed. New York: J. Wiley e Sons, 1956. 2 v.
HUSCH, B.; MILLER, C. I.; BEERS, T. W. Forest mensuration. 3.ed. New York: J. Wiley e Sons, 1982. 402 p.

KMENTA, J. Elementos de econometria. São Paulo: Atlas, 1978. 670p.

PÉLLICO NETTO, S. Amostragem em dois estágios com unidades primárias de tamanhos diferentes. Ciência Florestal, Santa Maria, v. 6, n. 1, p. 147-155, 1996.

PÉlliCO NETTO, S.; BRENA, D. A. Inventário florestal. Curitiba: Ed. dos Autores, 1997.316 p.

SUKHATME, P. V. et al. Sampling theory of survey with applications. Ames: Iowa State College Press, 1984. 526 p.

YAMANE, T. Elementary sampling theory. Englewood Cliffs: New York University/Department of Economics: Prentice-Hall, 1967. 405 p. 